\title{
Fighting cheaters: How and how much to invest
}

\author{
JUAN CARLOS NUÑO ${ }^{1}$, MIGUEL ÁNGEL HERRERO ${ }^{2}$ \\ and MARIO PRIMICERIO \\ ${ }^{1}$ Departmento de Matemática Aplicada a los Recursos Naturales, ETSI de Montes, \\ Universidad Politécnica de Madrid. 28040-Madrid. Spain \\ email: juancarlos nuno@upm .es \\ ${ }^{2}$ Institute for Interdisciplinary Mathematics and Departamento de Matematica Aplicada. Facultad de Ciencias \\ Matematicas, Uitiversidad Complateise de Madrid, 28040 Madrid. Spain \\ ${ }^{3}$ Dipartimento di Matematica Ulisse Dini', Universita degli Studi di Firenze. 50134-Firenze. Italy
}

\begin{abstract}
Human societies are formed by different socio-economical classes which are characterized by their contribution to, and their share of, the common wealth available. Cheaters, defined as those individuals that do not contribnte to the common wealth bnt beneht from it, have always existed, and are likely to be present in all societies in the foreseeable fnture. Their existence brings about serious problems since they act as sinks for the commnnity wealth and deplete resources which are always limited aud often scarce.

To fight cheaters, a society can invest additional resources to pursue one or several aims. For instance, an improvement iu social solidarity (e.g. by fostering educatiou) may be sought. Alternatively, deterrence (e.g. by increasing police budget) may be enhanced. Then the following questions naturally arise: (i) how much to spend and (ii) how to allocate the expenditure between both strategies above. This paper addresses this general issue in a simplified setting, which however we believe of some interest. More precisely, we consider a society constitnted by two prodnctive classes and an unprodnctive one, the cheaters, and proposes a dynamical system that describes their evolution in time. We find it convenient to formulate our model as a three-dimensional ordinary diflerential equation (ODE) system whose variables are the cheater population, the total wealth aud oue of the productive social classes. The statiouary values of the cheater populatiou aud the total wealth are studied in terms of the two parameters: $\phi$ (how much to invest) and $s$ (how to distribute such expenditurej. We show that it is not possible to simultaueously miuimize the cheater population and maximize the total wealth with respect to $\phi$ and $s$. We then discuss the possibility of defining a compromise function to find suitable values of $\phi$ and $s$ that optimize the response to cheating. In our opinion, this qualitative approach may be of some help to plan and implement social strategies against cheating.
\end{abstract}

\section{Introduction}

Human societies are very complex socio-economical systems whose members are expected to contribute to, and benefit from, a collectively generated pool of resources that we shall term as the common wealth. However, a subclass of the population (the cheaters) provide little, if any, contribution to the common wealth but at the same time take advantage of 
any welfare that society provides to its members. Cheaters are known to be present in any human society to this day, and since their contribution to the community is merely a negative one, the need arises of keeping their members as low as possible. This last in turn further depletes social resources in order to support policies aimed at controlling such social group.

Concerning control measures, one can roughly distinguish two different strategies: (A) to foster education and social awareness, and (B) to pursue cheaters by police forces. Concerning (A), it is widely assumed that improving cultural and educational facilities results in an easier promotion from low-income to high-income classes, which in turn is expected to yield a high proportion of law-abiding, fairly contributing taxpayers. However, it should be noticed that upper classes (dehned in terms of their income) develop their own ways of cheating (e.g. tax evasion), whose global impact on society may be even higher than that produced by similar attitudes in low-income classes. On the other hand, the need of a police force to check criminality, which can be considered as a particularly important aspect of cheating, is beyond discussion. This fact notwithstanding, maintaining an efficient police force requires of considerable resources. Clearly, implementing (A) and (B), or any suitable combination thereof, requires of adequate public spending. Thus, the following questions naturally arise:

(a) Given that the budget available is limited, which fraction of the total wealth should be devoted to fight cheating? It would be paradoxical indeed to spend more on that purpose than the actual losses generated by cheating itself. On the other hand, an insufficient effort in that direction may result in a social perception of immunity, while at the same time preventing such funds from being used for other social purposes.

(b) How should the appropriate budget be distributed anong strategies (A) and (B) above? Note that such choices are unlikely to be equivalent neither in terms of political clout nor in what concerns estimated costs of measures required to implement them Moreover, the choice of a distribution among (A) and (B) is not independent of the extra expenditure. In other words, the efficiency of this allocation depends on the budget.

(c) Which ultimate goal should one look for: to keep cheaters at their lower possible level, or to ensure the higher possible global wealth? As it turns out, both aims may not be compatible, since a low cheater population might be only kept at the price of a huge. maybe unaffordable, economical effort. A reasonable compromise, defined in terms of a suitable pay-of function, could be preferred by decision makers.

In an attempt to shed light on these questions, we shall discuss in this work a simple mathematical model, which however displays some of the features recalled above. It can be considered in the same spirit as that presented in [17]. where a criminal-prone selfprotected society divided into $n$ diflerent socio-economical classes (defined in terms of their contribution to the total wealth) was considered. As in [17], we shall deal with a system of ordinary differential equations (ODEs), thus assuming continuous variables (e.g. relative population of each species) that depend on time. This approach seems appropriate to model the dynamics of large human populations, where births and deaths take part nearly continuously. This is in sharp contrast with other types of populations, where growth occurs in discrete generations, whence models based on Difference Equations should be more adequate (see, for instance, [14]). 
Since we shall consider ODEs models, we will assume that the populations under consideration are homogeneous in space. While we intend to include space dependence in future work, we retain the space homogeneous case to be of some interest on its own. as we will explain below. Also, our model is a deterministic one, and it therefore ignores random fluctuations in the dynamics considered. A feature that we will deal with herein is strategy optimization, so that quantitative arguments to asses the comparative weight of strategies (A) and (B) above on policy making will be discussed.

As to the preceding to this work, we should mention Gary S. Becker's seminal paper [3] (see also [4]), where several economical aspects related to crime, e.g. police, punishment, employment, schooling and training programs, are explicitly considered, and the search of a trade-off between the crime-induced costs and resources to check it is proposed. This economic approach has been later pursued by other authors (see, for instance, [9]).

This paper is organized as follows: Section 2 contains a brief description of population models relevant to our forthcoming analysis. The particular two-class model that we will discuss in detail herein is presented in Section 3. Section 4 is concerned with an optimization problem associated to dynamical system dehined in the previous section. A sensitivity analysis is carried out in Section 5 to check the robustness of the model. Finally, we discuss on the results obtained and on possible extensions thereof that could incorporate more general social situations. In order to keep the flow of the main arguments in this work we have conhined to appendices some technical (although simple) details.

\section{A population dynamics approach}

Population models have fostered a huge amount of mathematical research in the last two centuries. For instance, in the nineteenth century, two famous mathematical models were brought about in an attempt to understand the evolution of human populations. We are referring to the Malthus law and the Verlhust or logistic equation (see, for instance. [21]). A second great burst of mathematical modelling occurred at the beginning of the twentieth century with the publication of the so called Lotka-Volterra equations to study predatorprey systems (a good introduction to these models can be found in [12,14]). Nonetheless, despite the relevance of population models in the natural sciences, their application in sociology and, in particular to criminality, seems to have been comparatively scarce. Only in recent years, models based on population dynamics have been proposed in this held. In this framework, a typical example can be roughly described as follows: a closed society in which spatial differences are neglected is assumed to be composed by different classes of individuals characterized by their attitudes towards illegal behaviour. The influences among the different classes, the flow from one class to another, etc. are then postulated in terns of kinetic terms, and the corresponding social dynamics is studied by analysing the solutions of the system of ODEs thus obtained.

To be more specific, we briefly describe a few examples of population nodels applied to study criminal behaviour. Recently, Paul Ormerod and his collaborators used this approach to measure how crime evolves over time in terms of socio-economical factors, as a consequence of agent interactions $[2,6,18]$. In it simplest form, the population is divided into three groups according to the propensity to commit crimes: non-susceptible, susceptible and hard-core criminals. The corresponding mathematical model accounts for 
the main factors that drive the flux among these classes (basically, demography, overall social and economical conditions, as well as the deterrence effect of the justice system). The values of the parameters entering the model are calibrated with actual data for a given class of crimes in a given region, so that not only the existing data are htted, but the results of the simulations can suggest how the society will react to particular changes in socioeconomical factors (e.g. increasing deterrence or increasing the imprisonment period). In latter versions of the model other classes are considered: prisoners [6] and recovered individuals' (the analogy with epidemiological models is evident) [25]. In the latter, the subpopulation of recovered criminals is subsequently split between non-susceptible and susceptible, and additional non-linear effects are taken into account.

Other models are much closer to the ecological framework and introduce phenomena similar to biological interactions as symbiosis, competition or predator-prey behaviours. For instance in [1] the 'symbiosis' between drug smugglers and drug consumers is considered. The Poincare-Bendixon theorem is then used to exclude the existence of limit cycles, and the influence of the parameters of the model on the equilibria of the dynamical system is studied. Predator-prey models have been proposed to describe the interactions between policemen (predators) and criminals (preys) [22]. The classical Lotka-Volterra model is then modified to include induced changes due to, for instance, policy measures or law enforcement.

An additional level of predation is studied in the 'triangular' model [16]. Criminals are predators with respect to the law-abiding people and are preys of guards; but, at the same time, guards have an impact on normal people that have to bear the cost of their salaries, equipment and facilities. The kind of predation guards exert on owners is basically of economical character, and indirectly affects the owners population. Unfortunately, the consideration of these three classes makes the dynamics much more complex and prevents a complete study, though some results can be obtained for particular cases (to explore further this model a mathematica demonstration can be found in [19]).

The population models previously presented, which are reminiscent of predator-prey models in ecology, assume that criminal activities act directly on victims and thus modify its population. However, most of the oflenses committed in our societies are against property, causing the depletion of individual wealth and, consequently, of the wealth of the whole society. In fact, modern societies not only care about criminal activities (that induce insecurity feelings) but also about other kind of antisocial behaviours as tax evasion, i.e. the existence of people that do not contribute to a common wealth. The presence of cheaters has been pointed out as one of the causes that prevent cooperation [11].

A special problem in this framework has been tackled in a previous paper [17] where a model of a criminal-prone society divided into $n$ classes $I_{i}$ according to their contribution to the total wealth $W$ was defined. It was therein assumed that individuals can move from one class to another depending on the total wealth of the society. The rates of social promotion from class $i-1$ to class $i$ and social relegation from class $i$ to $i-1$ are given by positive real parameters $\alpha_{i-1}$ and $\beta_{i}$, respectively. Criminals are considered as a class apart, and the same happens to a specialized class of guards that is hired by the society to control criminality. More precisely, let us denote the population of each class at time $t$ by $X_{i}(t) i=1, \ldots, n$. The criminal and guard populations at time $t$ are given by $Y(t)$ and 
$G(t)$, respectively. We assume the total population of the society to be constant, i.e.

$$
\sum_{i=1} X_{i}(t)+Y(t)+G(t)=N .
$$

At time $t$, each class contributes to the total wealth $W(t)$ (measured in terms of budget) proportionally to its population:

$$
W(t)=\sum_{i}^{n} c_{i} X_{i}(t)
$$

where, for instance, $c_{i} \leqslant c_{i+1}$ for all $i=1,2, \ldots, n-1$ and $c_{1} \geqslant 0$. For simplicity, it is also assumed that guards and criminals do not contribute to the legal economy.

In a free-of-criminals society (where police is also absent) the flow of individuals through the social classes is given by

$$
\dot{X}_{i}(t)=\alpha_{i-1} X_{i-1}(t)-\left(\alpha_{i}+\beta_{i}\right) X_{i}(t)+\beta_{i+1} X_{i+1}(t)
$$

We take, by delinition, $\alpha_{n}=\alpha_{n}=\beta_{1}=\beta_{n+1}=0$. It is then straightforward to see that the equilibrium distribution of individuals among different classes satishies:

$$
\begin{array}{rlr}
-\alpha_{1} X_{1}+\beta_{2} X_{2} & =0, \\
\alpha_{i-1} X_{i-1}-\left(\alpha_{i}+\beta_{i}\right) X_{i}+\beta_{i+1} X_{i+1} & =0 \\
\alpha_{n-1} X_{n-1}-\beta_{n} X_{n} & =0 .
\end{array} \quad \text { for } \quad i=2, \ldots, n-1,
$$

Besides, for any $t$

$$
X_{1}(t)+X_{2}(t)+\cdots+X_{n-1}(t)+X_{n}(t)=N,
$$

where $N$ is the whole population. In general this is a non-linear system since the kinetic parameters $\alpha_{i}$ and $\beta_{i}$ may depend on $X_{1}, \ldots, X_{n}$ through $W$. It can be proved (see Appendix A) that, under general assumptions, at least one non-trivial equilibrium point exists.

To take into account the presence of criminals $Y$, such scheme has to be modilied to include the effect they cause to the distribution of the population, as well as the damage caused to the total wealth A new ODE nust be introduced to describe the time evolution of $Y$ which depends on $W$ and on some of the other classes $X_{i}$. The presence of guards $G$ could also be taken into account by introducing an additional ODE for the evolution of their number, or simply by assuning that $G$ is given but its value affects the equation of $W$ (the cost of maintaining a police) in addition to the equation of $Y$ (see [17] for details).

The mathematical formulation of this kind of models depends on the question to be addressed. In [17] we considered explicitly the effect of crimes in the total wealth and how its impact can be controlled by the society. It was proved that this type of systems are criminal-prone, in the sense that criminal-free steady states are unstable under small perturbations in the socio-economical context. Moreover, we compared two different strategies (increasing police forces versus increasing social measures) to fight criminality (see [17] for further details). In the next section, we present a particular $W$-model with only two social classes that allow us to go a step further in the study of criminality and how to control it. 


\section{Two classes $\boldsymbol{W}$-model}

Models in which the total population wealth $W(t)$ is considered as a variable, are particularly useful to tackle the questions stated at the introduction and we shall refer to them as $\boldsymbol{W}$-models. We shall next address one such situation with only two social groups. defined according to their contribution to the common wealth $W$, say poor and rich and a class formed by individuals that do not contribute to $W$, the cheaters. For the sake of simplicity, it is assumed that cheaters are recruited just from the poor class and that when they decide not to cheat again (either naturally or by the action of social pressure) they go back to the same class.

Let $X_{1}(t), X_{2}(t)$ and $Y$ be the population of poor, rich and cheaters at time $t$, respectively. It is assumed that the total population remains constant, i.e.

$$
N=X_{1}(t)+X_{2}(t)+Y(t) .
$$

Let us start by considering a cheater-free society, so that

$$
\begin{aligned}
& \dot{X}_{1}=-\alpha X_{1}+\beta X_{2}, \\
& \dot{X}_{2}=\alpha X_{1}-\beta X_{2},
\end{aligned}
$$

where $\alpha$ and $\beta$ are the coefficients of social mobility (promotion and relegation, respectively).

With this definition of the rich class and taking into account the Pareto principle or $20-80$ rule (see, for instance, [20]), which establishes that $20 \%$ of the population can be considered rich, it is reasonable to select $\beta \approx \mathbf{4} \alpha$. On the other hand, the values of $\alpha$ and $\beta$ depend on the mobility we assume in the society. For definiteness, we choose one in which $\beta=0.04$ and $\alpha=0.01$. Of course, these values depend on the choice of the unit of time. If we assume this unit to be 1 year then about $5 \%$ of the population changes it social status in 1 year.

These parameters can also depend on the total wealth, as we assumed in [17], because social mobility is sensible to $W$. Here we will assume, in particular, that parameter $\alpha$ may increase as a consequence of the social policy adopted.

\subsection{Equation for the total wealth, $W$}

The total budget of the society changes over time because of social mobility. This can be described by the following differential equation:

$$
\dot{W}=P-E-(S+G),
$$

where $P, E, S$ and $G$ will be defined below. To begin with, $P$ is the rate of wealth production. As stated in the previous section, it is quite natural to assume a linear dependence on $X_{1}$ and $X_{2}$ :

$$
P=c_{1} X_{1}+c_{2} X_{2} .
$$

It is convenient to normalize wealth with respect to $c_{1} N$ (i.e. the wealth produced, or the taxes paid, per year by a population in which all the individuals belong to the poor class) 
and to normalize $X_{1}$ and $X_{2}$ to the total population $\left(z=\frac{X_{1}}{N}\right.$ and $\left.u=\frac{X_{2}}{N}\right)$. Thus

$$
\frac{P}{c_{1} N}=z+a u
$$

The value of constant $a$ now depends on the definition we take for rich and poor classes. To be specific we assume this definition in agreenent with the $20 / 80$ rule (see [20]) that states that $20 \%$ of the population owns $80 \%$ of the resources (and hopefully contributes up to the $80 \%$ of the hisal income). This neans that we will take $a=16$.

The term $E$ is the rate of current expenses (neither related nor influenced by cheating). It is natural to assume that they represent a given fraction of the actual total wealth $W$, so that

$$
E=\theta W .
$$

Modern societies tends to adjust its expenses to the GNP so $\theta$ should be close to 1 . For simplicity, we will take $\theta=1$ in the sequel.

The next term $S+G$ represents the additional expenditure per unit time (i.e. 1 year) in order to counteract the growth of cheating. Their sum will be a fraction $\phi$ of the total wealth. This quantity $\phi$ must be split into two parts: expenses aimed at social promotion (represented by $S$ ) and those devoted to crime repression (represented by $G$ ). We shall write

$$
\begin{aligned}
& S=s \phi W, \\
& G=(1-s) \phi W,
\end{aligned}
$$

where $s \in[0,1]$. Thus, $\phi$ measures how much a community spends to hight cheating whereas $s$ measures how it spends this amount. In principle, $\phi$ would depend on $Y$, although here it will be considered as a constant. A motivation for that choice is that in a quasi-stationary approach it is reasonable to assume that the change of political strategy has a time of reaction that is long with respect to characteristic times of social evolution. This implies that the system always relaxes to a stationary regime between any two consecutive changes of the control parameters $\phi$ and $s$.

Finally, normalizing $u=\frac{X_{2}}{N}, z=\frac{X_{1}}{N}$ and $w=\frac{W}{N c_{1}}$, equation (3.3) reduces to

$$
\dot{w}=z+16 u-(1+\phi) x \text {. }
$$

\subsection{Equation for the cheater population, $Y$}

Arguing as in [17], the time evolution of the cheater population can be written as

$$
\dot{Y}=R-D-A,
$$

where $R, D$ and $A$ represent recruitment, natural decay and arrests respectively, always referred to the unit of time ( 1 year). For simplicity, we assume that recruitment comes only from the poor class and that it is a linear function of $Y$, proceeding according to some contagion process:

$$
R=\left(\tau_{0}(w)+\tau_{1}(w) Y\right) X_{1}
$$


For $\tau_{0}$ and $\tau_{1}$ we propose the following choice:

$$
\tau_{i}=\bar{\tau}_{i} \frac{1}{1+\eta_{i} s \phi w_{i}}
$$

for $i=0,1$, according to which an increase in $w$ translates into a reduction of the rate of recruitment.

In the case of constant $\tau_{0}$ and $\tau_{1}$, i.e. $\eta_{0}=\eta_{1}=0$, we will assume $\tau_{0}=10^{-2}$ and $\tau_{1}=10^{-1}$ so that in case of having a cheater population $Y$ of the order of $10^{-1}$ both terms in $(3.10)$ be of the same order.

Concerning $D$ we assume as usual a decay proportional to the population and that about $1 \%$ of the cheater population leaves that class in one year:

$$
D=\mu Y
$$

so that a reasonable approximation is $\mu=10^{-2}$.

The last term $A$ measures the removal of cheaters by the action of guards. We assume that it is proportional to the resources invested in this matter, thus

$$
A=k(1-s) \phi w Y
$$

Here $k$ represents the efficiency of a policeman (the average fraction of cheaters captured in one year) divided by the equivalent yearly-normalized cost. Notice that a stationary value for $w$ in a cheater-free society ranges from 1 (all poor) to 16 (everybody is rich). Moreover, a reasonable assumption for the extra expenditure is $\phi=10^{-2}$. So, taking $k=1$ means that $1 \%$ of the criminals are caught in 1 year for intermediate values of $s$.

To complete the model we have to define the dependence of $\alpha$ and $\beta$ in (3.2) on the policy choice for fighting cheating. For simplicity, we assume that $\beta$ remains constant (say $\beta=0.04$ ) and that $\alpha$ can increase due to the amount of money devoted to social measures:

$$
\alpha=\alpha_{0}+\alpha_{1} s \phi w .
$$

For definiteness, we will take $\alpha_{0}=0.01$ as before and $\alpha_{1}=1$.

Setting $y=\frac{Y}{N}$ and considering the constraint $u+z+y=1$ (cf. (2.1)), the dynamical system we will analyse in the next section is given by

$$
\left.\begin{array}{l}
\dot{z}=-(0.01+s \phi w) z+0.04(1-z-y)-\dot{y} \\
\dot{y}=(0.01+0.1 y) z-0.01 y-(1-s) \phi w y \\
\dot{w}=z+16(1-z-y)-(1+\phi) w .
\end{array}\right\}
$$

In Appendix B, we present a related but simpler model with only two classes, taxpayer and cheaters, that in spite of its simplicity exhibits an interesting behaviour.

Before going any further, let us find what is the largest wealth that this society can produce. It is natural to expect that this will be achieved in the absence of cheaters and when the richest class takes its largest possible value. A free-of-cheaters society is possible only if they can not be recruited from the poor class, i.e. $\tau_{0}=\tau_{1}=0$. In addition, social promotion is enhanced as much as possible when public expenses are completely devoted 
to that end, i.e. $s=1$. In this case, $\bar{y}=0$ and the total wealth reads

$$
\bar{w}^{\prime}=\frac{-1+319 \phi+\sqrt{102081 \phi^{2}-318 \phi+1}}{40 \phi(1+\phi)} .
$$

The maximun value of the total wealth turns out to be $\bar{w}_{\max } \approx 14.5$ and it is attained for $\phi=\frac{53}{34027}+\frac{1276}{102081 \sqrt{15}} \approx 0.05$.

When both $\tau_{0}$ and $\tau_{1}$ are different from zero as in (3.15), then a positive cheater population appears. If no additional expenses are devoted to fight cheating, i.e. $\phi=0$, then the qualitative analysis of system (3.15) shows that the stationary total wealth is drastically reduced to $\bar{w}_{0} \approx 0.45$. This is about a thirty times drop if compared with $\bar{w}_{\text {max }}$. The corresponding criminal population reaches $\bar{y} \approx 0.89$. This means that almost $90 \%$ of the population do not pay taxes (cheaters). However, as we will see in the next section, the losses in the total wealth can be reduced by increasing public expenses $\phi$. Nonetheless, this should be done in an appropriate way, since, in an attempt to completely eliminate the criminal population, the total wealth could be depleted even more than its local minimum $\bar{w}_{0}$ (in fact, $\bar{w}$ tends to 0 when $\phi \rightarrow \infty$ ).

\section{Qualitative analysis}

In this section we analyse the equilibrium properties of the dynamical system $(3.15)$ in terms of the control parameters $\phi$ (how much) and $s$ (how). The equilibrium level of cheaters and the total wealth of the society can be determined by setting the right-hand side of (3.15) equal to zero. The resulting algebraic system is non-linear and can not be solved analytically in general. A numerical solution can still be found using standard packages as, for instance, MAPLE. The stationary cheater population turns out to be the solution of a fourth-degree equation. Then the corresponding total wealth can be in turn obtained from it (see Appendix C for further details).

Figures 1 and 2 show the three-dinensional plot for both the cheater population and the total wealth at equilibrium as a function of $\phi$ and $s$. As it can be seen, while the cheater population $\bar{y}$ decreases monotonically with $\phi$ for all values of $s$, the total wealth $\bar{w}$ presents, for all $s$, a peak for a definite value of $\phi$.

The way this peak depends on $s$ is not straightforward. As $s$ tends to 0 it is displaced to larger values of $\phi$. Nonetheless, for $s=0$ the maximum is attained for a lower value of $\phi$ (see Figure 3). Looking at the other extreme case $s=1$, the displacement is neither monotonous: As $s \rightarrow 1$, the peak moves again to larger values of $\phi$.

These results suggest that to attain the lowest possible level of cheaters, authorities should increase as much as possible the public spending $\phi$. However, this would cause the depletion of the common pool to unacceptable levels. A possible goal would be to maintain the total wealth in its maximum for a couple $(\phi, s)$. However, this would force the society to live with a high level of cheaters, which may prove to be unsustainable. As stated previously, the question arises about how to get a compromise between these two scenarios.

A standard way of addressing this matter is to define a new compromise function from the equilibrium solutions obtained from the dynamical system (3.15). However, the choice 


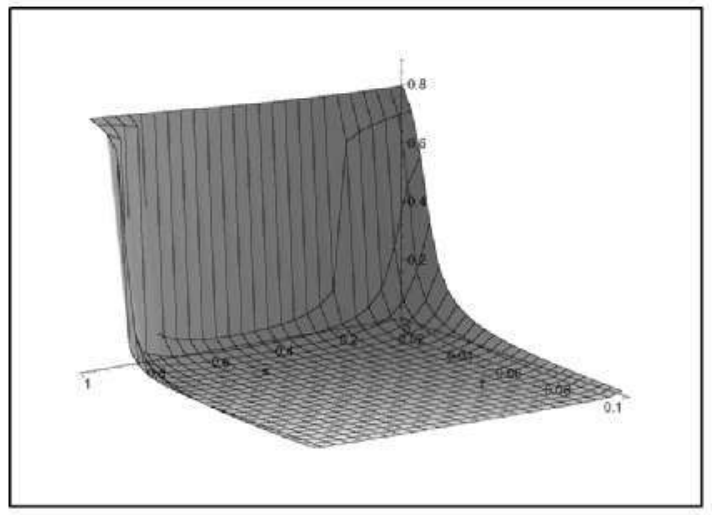

FIGURE 1. Stationary cheater population as a function of $s$ and $\phi$. The rest of the parameter values are defined in the main text.

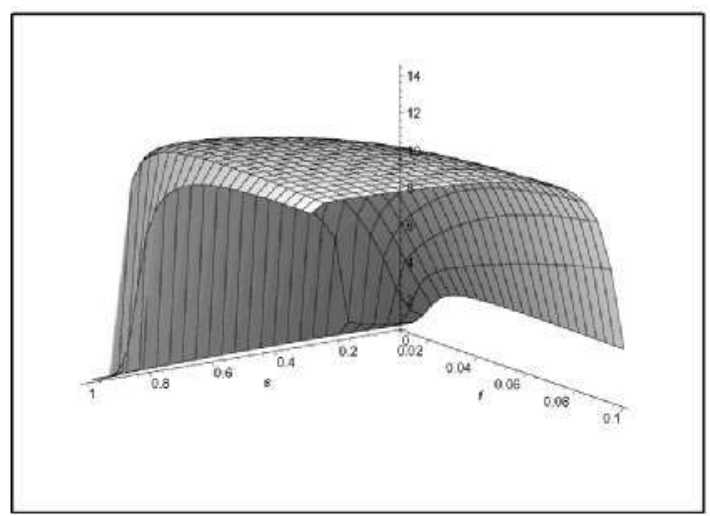

FIGURE 2. The corresponding stationary total wealth as a function of $s$ and $\phi$. The rest of the parameter values are defined in the main text.

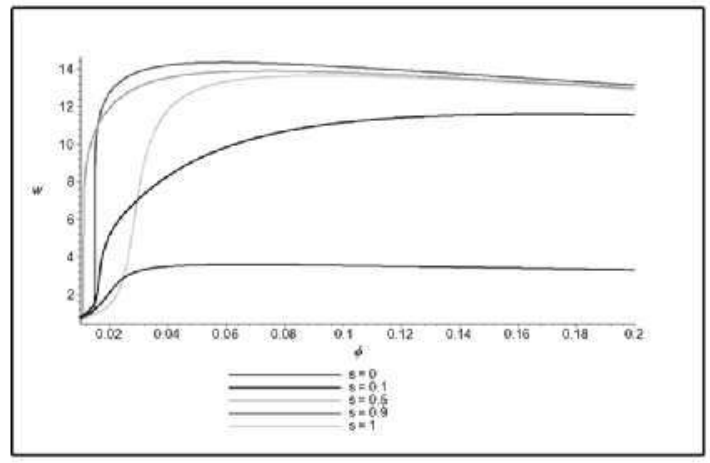

FIGURE 3. The stationary common wealth as a function of $\phi$ for different values of $s$. As can be seen, all curves present a peak for a value of $\phi$ that depends on $s$. All curves approach the horizontal axis as $\phi$ tends to infinity. The rest of parameter values are as defined in the main text. 


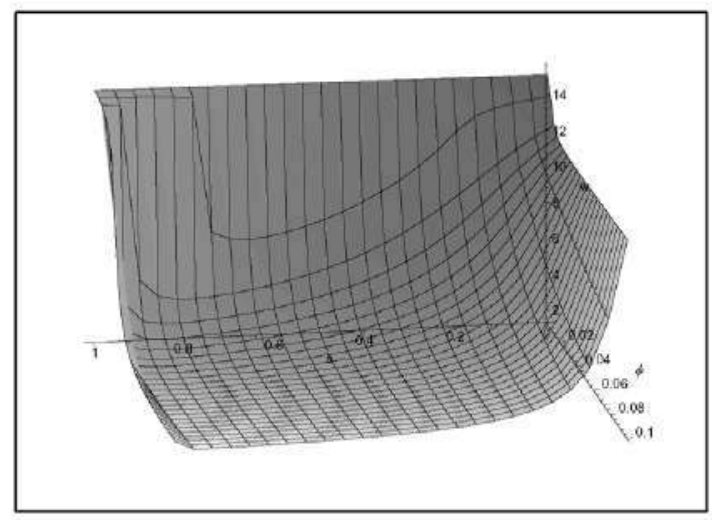

Figure 4. Three- dimensional plot of the stationary compromise function $\bar{L}$ in terms of $\phi$ and $s$. As can be seen, $\bar{L}$ reaches an absolute minimum at $(\phi \approx 0.05, s \approx 0.9)$. Parameter setup as in previous figures.

of such a function is not immediate. For instance, the ratio

$$
\bar{R}=\frac{\bar{w}}{\bar{y}}
$$

that is, the stationary total wealth per cheater, can be shown to attain an absolute maximum but for a quite large value of $\phi$. This means that to achieve the largest possible total wealth per cheater ordinary expenses should be largely increased (about three times per year) which for practical purposes is unacceptable.

An alternative approach is to define a set of compromise functions each of them representing a certain distance to an 'ideal' and unreachable point where the two criteria are simultaneously optimized. This idea is behind the so called compromise programming, introduced by Yu and Zeleny ( $[23,24])$. For instance, we can use the Euclidean distance to define

$$
\bar{L}=\sqrt{(\bar{w}-16)^{2}+\lambda \bar{y}^{2}} .
$$

Here $\lambda>0$ is a normalization factor that allows to weight each criteria differently. Since $\bar{L}$ is an implicit function of the two parameters $s$ and $\phi, \bar{L}$ represents the distance from a surface, defined by the functional dependence between $\bar{w}$ and $\bar{y}$ as a function of $(s, \phi)$, to the 'ideal' line $(1-\bar{y}, \bar{w})=(1,16)$. Figure 4 depicts a three-dimensional plot of $\bar{L}$ as a function of $s$ and $\phi$ for $\lambda=1$. As can be seen, in this range the surface decreases sharply with $\phi$ until it reaches a plateau. An absolute minimum exists for $s \approx 0.9$ and $\phi \approx 0.05$. Moreover, these values are quite insensitive with respect to $\lambda$.

Finally, a related possibility to be considered is to adjust $\phi$ to maximize the total wealth, knowing that the cheater population sharply decreases with $\phi$. As a matter of fact, for the couple $(0.05,0.9)$ that approximately maximizes the common wealth, $\bar{w}_{\max } \approx 14.5$, the cheater population is already around $1 \%$ of the population, i.e. $\bar{y} \approx 0.01$ (see figures 1 and 2).

At this juncture, it is worth noting that a more general optimization approach to fight cheaters consists controlling the system through the parameters $\phi$ and $s$ during all its 


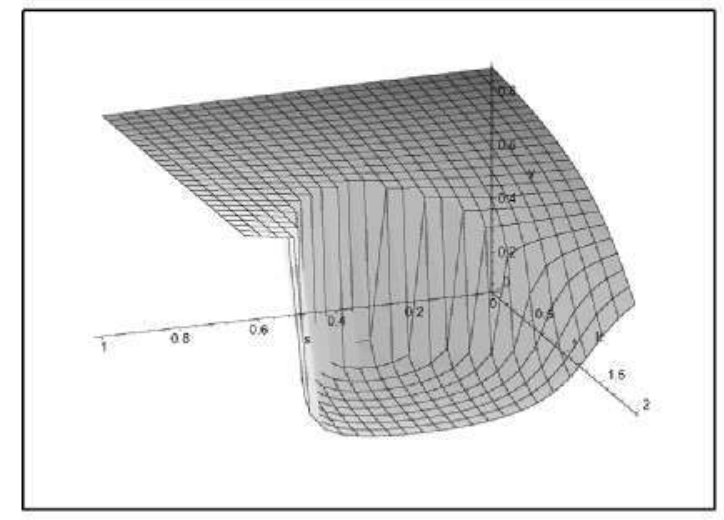

Figure 5. Cheater population $\bar{y}$ as a function of $k$ and $s$. As is depicted in Figure 5, some of the level curves are not continuous as appear here. Parameter values are: $\phi=0.01 ; \alpha_{0}=0.01 ; \alpha_{1}=1$; $\mu=0.01 ; a=16 ; \theta=1 ; \tau_{1}=0.1 ; \tau_{0}=0.01 ; \beta=0.04$.

evolution in a given time interval $\left(0, T_{f i n}\right)$ and not just at its equilibrium states. For this purpose, an appropriate cost functional should be defined in terms of parameters $\phi$ and $s$, that can also be assumed to be time-dependent and subject to appropriate requirements. A natural choice for such functional would be an integral function over a time interval $\left(0, T_{f i n}\right)$ of a linear combination of $1 / w(t)$ and $y(t)$. Alternatively , the function $L(t)$ defined as in (4.2), but with $\bar{w}$ and $\bar{y}$ replaced by $w(t)$ and $y(t)$, can be considered. However, a thorough analysis of that type of problems (which would be in the spirit of $[10,11]$ ) is beyond the scope of this article, and we intend to address it elsewhere.

\section{Sensitivity on $k$}

Besides the control parameters $\phi$ and $s$, the relationship between the parameter $k$, that measures the efficiency of police repression (see (3.13)), and $\alpha_{1}$, the efficiency of public spending to foster social promotion (see (3.14)), play a fundamental role in the system. In this section, we study how sensitive the system to variations in $k$. Let us take $\alpha_{1}=1$ and let $k$ be the second control parameter, together with $s$, instead of the extra expenditure that is now fixed to $\phi=10^{-2}$.

Figure 5 depicts a three-dimensional plot of the cheater population as a function of $s$ and $k$. As it can be seen, level curves for fixed $k$ presents a minimum as a function of $s$ only when $k$ is larger than a critical threshold, $k_{c} \approx 1.25$. Below it, there is no minimum with respect to $s$ and the lowest level of criminality is attained either for $s=1$ if $k<0.3$ or for $s=0$ for $0.3<k<k_{c}$.

The transition from a higher level of cheaters to a lower one is observed better in Figure 6, where several projections for different values of $s$ are depicted. Transitions become sharper as $s$ increases towards 1 . Nonetheless, if $s=1$ the cheater population does not depend on $k(\bar{y} \approx 0.87)$. When projections are on $k$, i.e. $s$ is left to vary for fixed values of $k$, a striking result is obtained as shown in Figure 7. When $k$ is less than a critical threshold $k_{c} \approx 1.24$ a multi-stability regime appears for intermediate values of $s$; a lowest level of cheater population coexists with a higher one for a range of $s$ that 


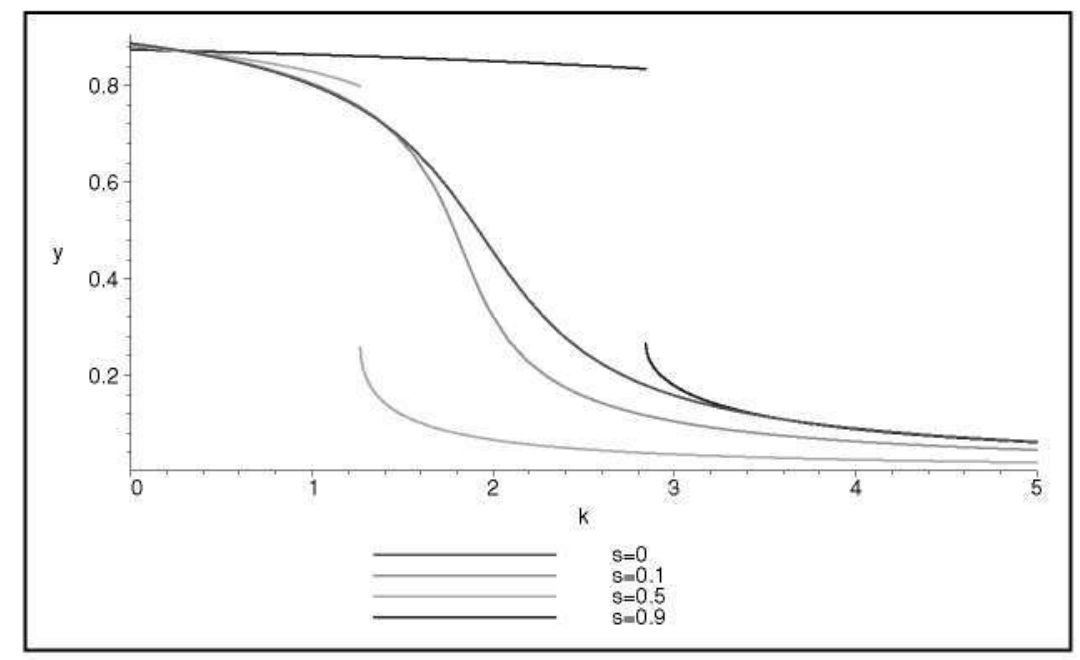

Figure 6. Projections of the cheater population as a function of $k$ for fixed values of $s$. Notice the discontinuities in the solution curves for certain values of $s$. The rest of the parameters take the values as in Figure 5.

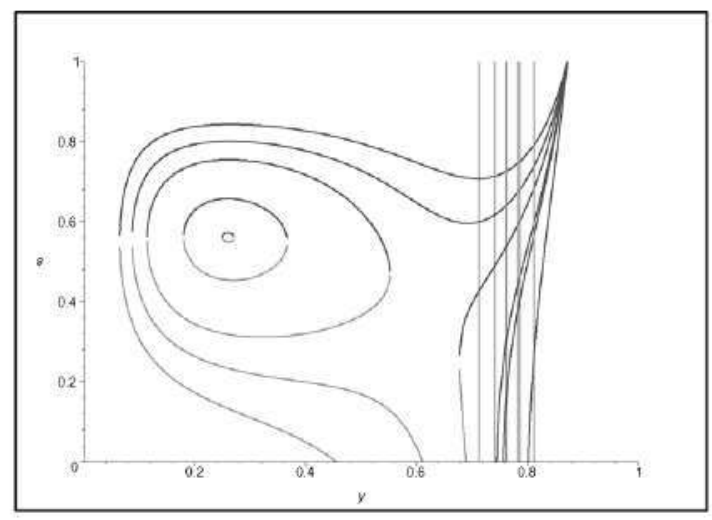

Figure 7. Cheater population as a function of $s$ for different values of $k$. Notice that the representation is inverted: $\bar{y}$ is now in the horizontal axis. As can be seen, for values of $k>k_{c}$ a bi-stability situation exists. Discontinuities are due to numerical resolution. Parameter values are as in the previous figures.

depends on $k$. As $k$ increases this multi-stability disappears and the cheater population reaches a plateau $\bar{y} \approx 0.04$ in the whole $s$-interval $[0,1]$. It is important to remark the abrupt increase near $s=1$ because for $s=1$ the level of cheaters is independent of $k$ and is approximately given by $\bar{y}=0.87$.

The total wealth behaves in a similar way, though the corresponding thresholds are different (see Figure 8). Below $k_{c} \approx 0.6, W$ increases monotonically with $s$. If $k>k_{c}$, then $W$ reaches a maximum for a particular value $s \in(0,1)$. 


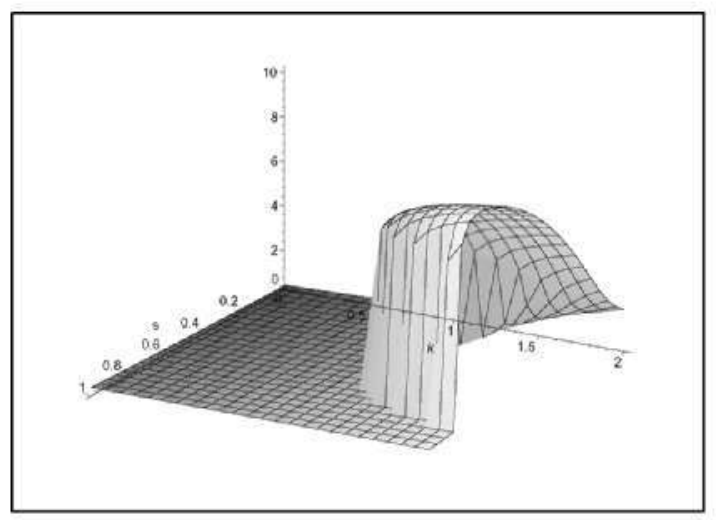

Figure 8. Three-dimentional plot of the total wealth $\bar{w}$ as a function of $k$ and $s$. Notice the sharp transition to large values when increasing $k$ that coincides with an abrupt drop in the level of cheaters. This threshold can be shown to be a function of $s$. Parameter values are as in the previous figures.

\section{Discussion}

The origin of cooperation in human societies is a fundamental open question $[5,11,13]$. One of the main challenges we face is to understand the existence of stable societies even in the presence of cheaters, that is, individuals that do not cooperate with but take advantage from the group. In pure selective terms, cheaters are better adapted and so they finally would ruin the society (a society full of cheaters is clearly not viable). To control antisocial behaviour, individuals have brought about different strategies, punishment being widely assumed to be the most efficient of all [7,8]. But punishment has a cost that could turn to be, in some situations, comparable to the damage produced by cheaters. It would be paradoxical to spend more money in defending ourselves against cheaters than that corresponding to the losses of having a percentage of the population behaving in an anti-social manner.

This paper has addressed this issue by using a simple mathematical model written in terms of differential equations. The model assumes that the dynamical system responds quickly enough to changes (either internal or external) to be almost permanently in a stationary state. So, the cheater population and the common wealth considered are equilibrium points of the dynamical system. Since, our system (3.15) has always an unique stationary state for the given parameter setup, then the equilibrium values of the cheater population $\bar{y}$ and the total wealth $\bar{w}$ are objective functions that can admit an optimal solution in terms of two parameters that represent how much to expend $(\phi)$ and how to invest this amount $(s)$. For the selected parameter setup, it turns out that whereas $\bar{w}$ has a maximun, $\bar{y}$ is a monotonically decreasing function of $\phi$ for all values of $s$. So, the question of what are the best values of $\phi$ and $s$ to choose can not be straightforwardly answered. The best for what? Either to maximize $\bar{w}$ or to minimize $\bar{y}$ ? As a matter of fact, to keep the level of cheaters arbitrarily small would require to use up the whole wealth of the society. As we said before, it does not seem a clever solution to spend all our resources to get such a payoff.

In a preliminary step, to solve this paradox we have studied several compromise functions in Section 4. The ratio between the common wealth and the level of cheaters 
through the function $\bar{R}$ has proven to give absolute maximun for $\phi \approx 2$ and $s=0.5$. This means that to reduce the cheater population keeping a wealthy society requires spending a non-realistic part of the yearly budget. An alternative might be to minimize the distance to an 'ideal point' where both, $\bar{w}$ and $1-\bar{y}$, reach their largest value $((1-\bar{y}, \bar{w})=(1,16)$. for the parameter setup used in Section 4). As a preliminary step, we have dehned a weighted Euclidean distance (4.2) that exhibits an absolute minimum for approximate values $s=0.9$ and $\phi=0.05$. These values are quite insensitive to variations in the normalization parameter $\lambda$. Finally, the possibility is suggested of selecting $\phi$ to obtain a signihicantly large value of $\bar{w}$ for which $\bar{y}$ is already low enough. In any case, we think that a point to be addressed here is that decision makers have to their disposal a kind of models to complement the empirical data they deal with. By considering several compromise functions that weight differently each aspect of the problem, authorities posses more elements to make an appropriate choice. A deeper comparison of the results obtained from different compromise functions is left as a future work.

Our results are not independent of the values of others parameters of the model. In fact, they are particularly sensitive to changes in $k$, the parameter that measures the efficiency of police actions. Obviously, if the efficiency of policing is much larger than that of social promotion, it would be beneficial to spend most of the money to enhance this strategy. On the contrary, if efficiency of guards is very low, money is always better spent in education and other social activities. A detailed study of the dependence of the level of criminality and the total wealth has been carried out in Section 4. As expected, a sharp and striking variation in these values occurs for a critical $k$-value.

Despite neglecting all stochastic factors, population models, that basically describe average dynamics, offer a useful approximation to the study of this kind of systems. Obviously, they are not able to predict neither individual behaviours nor explain energent behaviours from basic rules. Thus, this deterministic models must be complemented with other techniques that include a probabilistic formulation. Specially fruitful are the so called cellular automata models where local rules of evolution are considered. We are currently applying this kind of approach to incorporate the spatial dimension to this model. Indeed, it is interesting to study the eflect of having different strategies on both how much $(\phi)$ and how (s) in different societies that coexist in a given area. For instance, in the discrete case, agents can be in two states: contributing or not to the total wealth. The probability of changing from one state to another might depend on both local rules (how many neighbours belong to each state) and global ones, according to the total wealth of the system (for instance, an increase in wealth could favour to become a taxpayer). In addition to the own dynamics of this model, it would be relevant to investigate the appearance of dynamical boundaries among regions with different politics, as well as how the obtained patterns depend on $\phi$ and $s$. We intend to address such issues elsewhere.

\section{Acknowledgements}

This work has been partially supported by Italy-Spain Integrated Action HI2006-0026 as well as by project FIS2009-13690 del Ministerio de Ciencia e Innovación of the Spanish government. Part of this work was performed while the third author was visiting 
the Institute for Interdisciplinary Mathematics (IMI) at Universidad Complutense de Madrid.

\section{Appendix A}

Social mobility in the absence of criminal (cheaters) was analysed in Section 3. Even when both social promotion and relegation depend on the total wealth, system (2.4) present at least one equilibrium point. This can be proved straightforwardly by noticing that each $\bar{X}_{k}$ can be expressed in terms of $\bar{X}_{1}$, i.e

$$
\bar{X}_{k}=\frac{\alpha_{1}}{\beta_{2}} \frac{\alpha_{2}}{\beta_{3}} \ldots \frac{\alpha_{k-1}}{\beta_{k}} \bar{X}_{1} \equiv \gamma_{k-1}(\bar{W}) \bar{X}_{1}
$$

for all $k=2, \ldots, n$. Equation $(2.2)$ can be now written in terms of $X_{1}$ :

$$
\bar{W}=c_{1} \bar{X}_{1}+\sum_{k=2}^{n} c_{k} \gamma_{k-1}(\bar{W}) \bar{X}_{1} .
$$

This allows to express $\bar{X}_{1}$ as an explicit function of $\bar{W}$ :

$$
\bar{X}_{1}=\frac{\bar{W}}{c_{1}+\sum_{k=1}^{n} c_{k} \gamma_{k-1}(\bar{W})}
$$

Now, using the constant population constraint (2.5) we see that

$$
N=\frac{\bar{W}}{c_{1}+\sum_{i=2}^{n} c_{i} \gamma_{i-1}(\bar{W})}+\sum_{k=2}^{n} \gamma_{k-1}(\bar{W}) \frac{\bar{W}}{c_{1}+\sum_{i=2}^{n} c_{i} \gamma_{i-1}(\bar{W})}
$$

whence

$$
\bar{W}=N \frac{c_{1}+\sum_{i=2}^{n} c_{i} \gamma_{i-1}(\bar{W})}{1+\sum_{i=2}^{n} \gamma_{i-1}(\bar{W})} .
$$

To see that this equation always admits at least one solution, let us define the auxiliary function:

$$
G(\bar{W})=\bar{W}-N \frac{c_{1}+\sum_{i=2}^{n} c_{i} \gamma_{i-1}(\bar{W})}{1+\sum_{i=2}^{n} \gamma_{i-1}(\bar{W})} .
$$

Here $G$ is continuous in $\left[0, c_{n} N\right]$ and satisfies that

$$
G(0)<0, \quad G\left(c_{n} N\right)>0 .
$$


Therefore, the equation $G=0$ has at least one solution $\bar{W} \in\left(0, N c_{n}\right)$.

\section{Appendix B}

The main qualitative features of the model described in Section 3 are already found in a simpler situation when the social structure of the society is not taken into account. What we want to outline here is that, although such model is now simpler, we can derive from its analysis some general properties that are also found in the much richer system (3.15).

Let us assume then that the contributor class is reduced to a single population $X$ and let $Y$ be the cheater population. As before, we assume that the total population is kept constant, i.e. $X+Y=N$. The contribution of law-abiding citizens gives rise to a common wealth $W$ that is used to keep the society working and to persuade cheaters to pay taxes. So, the time evolution of the total wealth is then given by

$$
\dot{W}=a(N-Y)-\theta W-\phi W .
$$

Here $a$ is the contribution of the law-abiding population $X=N-Y$ and $\theta$ is the rate of expenditure that is required to maintain the society, and $\phi$ measures the extra cost that is devoted to fight cheaters.

The time evolution of the cheater population is given by

$$
\dot{Y}=\tau(W)(N-Y)-\gamma(W) Y,
$$

where $\tau$ is the rate of cheater recruitment of cheater from the normal population and $\alpha$ in the reduced version of (3.2) is the rate of transformation into law-abiding citizens. As in the two-dimensional model, how the extra expenditure is devoted to each of the measures is regulated by the authorities by tuning the parameter $s$. In particular, let us take $\tau_{1}=0$ in (3.10) and

$$
\tau_{0}=\frac{\tau_{0}}{1+\eta_{0} s \phi W}
$$

whereas $\gamma$ is selected to linearly depend on $W$ :

$$
\gamma=\mu+k(1-s) \phi W
$$

With these choices the dynamical system that describes the reduced system behaviour is

$$
\left.\begin{array}{rl}
\dot{Y} & =\frac{\overline{\tau_{0}}}{1+\eta_{0} s \phi W}(N-Y)-(\mu+k(1-s) \phi W) Y, \\
\dot{W} & =a(N-Y)-\theta W-\phi W .
\end{array}\right\}
$$

We next re-scaled variables by setting $y=\frac{Y}{N}$ and $w=\frac{W}{a N}$. Therefore,

$$
\left.\begin{array}{l}
y=\frac{\overline{\tau_{0}}}{1+\eta_{0} s \phi W}(1-y)-(\mu+k(1-s) \phi w) y, \\
\dot{w}=(1-y)-(\theta+\phi) w
\end{array}\right\}
$$




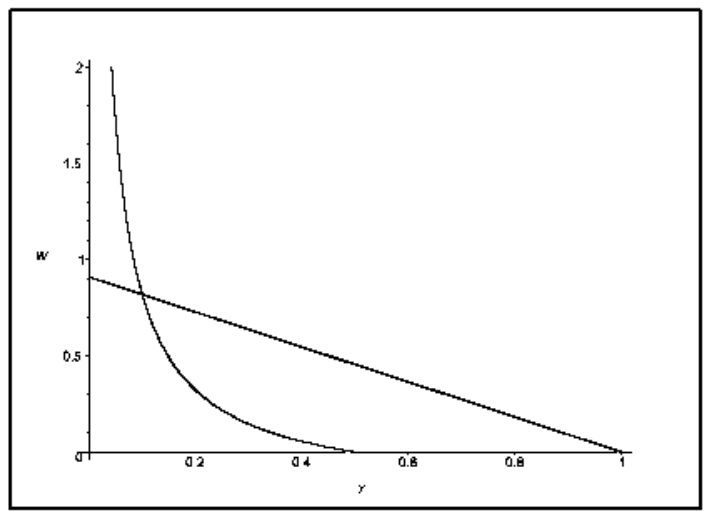

FIGURE B 1. Null and infinity isoclines. The parameter setup is the following: $\mu=0.01, k=1$, $\overline{\tau_{0}}=0.01, \eta_{0}=100$ and $0=1$.

where the system parameters have been redefined as follows: $\eta_{1}=\eta_{1} a N$ and $k=k N a$ (for simplicity we keep to the same notation).

Contrary to the two-class $W$-model, system (B 6) can be qualitatively studied by using bi-dimensional techniques. In particular, by analysing the isoclines we can prove the existence of a unique equilibrium point (see figure B 1). The horizontal (null slope) isocline is given by

$$
w=\frac{1-y}{\theta+\phi} .
$$

The vertical (infinite slope) isocline is implicitly defined by the equation

$$
\overline{\tau_{0}}(1-y)-\left(1+\eta_{0} s \phi w\right)(\mu+k(1-s) \phi w) y=0 .
$$

An equivalent parameter setup to that chosen in Section 3 is the following $\mu=0.01$, $k=1, \bar{\tau}_{0}=0.01, \eta_{0}=100$ and $\theta=1$. It can be easily seen that both isoclines cross only once inasmuch for $w=0 h$-isocline is over $v$-isocline, whereas the contrary occurs for $y=0$ where $h$-isocline is below the $v$-isocline. Besides, the first derivative of $w$ with respect to $y$ in (B 8) is always negative, irrespectively of the parameter values, whereas the second derivative is always positive. Then, for this case, the equilibrium point of (B 6) exists and is unique. In addition, this equilibrium point is always asymptotically stable.

Figure $\mathrm{B} 2$ depicts the three-dimensional plots of both the stationary cheater population and the corresponding total wealth as a function of $\phi$ (how much) and $s$ (how). As can be seen, whereas the cheater population decreases monotonically as $\phi$ increases for all $s \in[0,1]$, the total wealth presents a peak for a given value of $\phi$ that is a function of $s$. As we did in Section 4, auxiliary objective functions could be defined to find a couple $(\phi, s)$ that optimizes the strategy to fight cheaters. A deeper analysis of this kind of two-dimensional system is been currently performed [15]. 


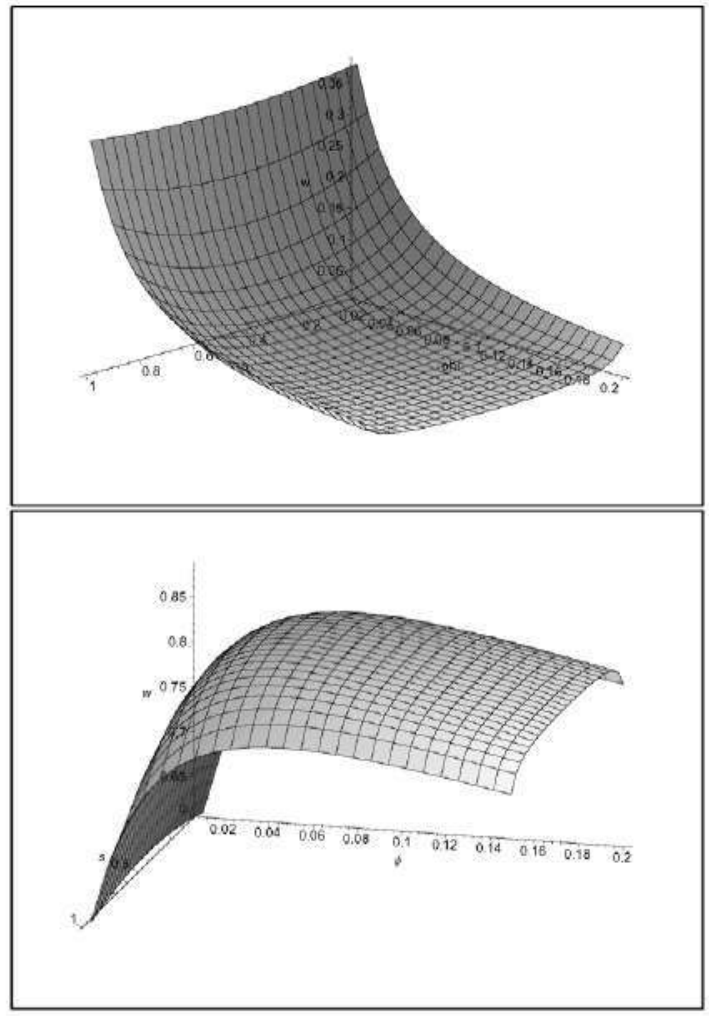

FigurE B 2. Cheater population and the corresponding total wealth for the 1 class model as a function of $\phi$ and $s$. The parameter setup is as in figure 9 .

\section{Appendix C}

This appendix gives an idea of the kind of algebraic equations tobe solved to obtain the equilibrium points of system (3.15). For the particular parameter setup selected, the stationary cheater population is the unique solution of the fourth-degree algebraic equation:

$$
\begin{aligned}
& 25610^{5} \phi^{2} s(s-1) \bar{y}^{4}+\left(40296000 s \phi^{2}-40800 \phi-43240000 \phi^{2} s^{2}+56000 \phi s\right. \\
& \left.\quad+2959600 \phi^{2}-400\right) \bar{y}^{3}+\left(29040 \phi-12121400 s \phi^{2}+15080000 \phi^{2} s^{2}-41400 \phi s\right. \\
& \left.\quad+270-2971230 \phi^{2}\right) \bar{y}^{2}+\left(-2565600 s \phi^{2}+2560000 \phi^{2} s^{2}+4071 \phi^{2}+71-5600 \phi s\right. \\
& \quad+4142 \phi) \bar{y}+4+4 \phi^{2}+8 \phi=0 .
\end{aligned}
$$

The corresponding stationary total wealth of the society is then the solution of the quadratic equation:

$$
20 \phi s(1+\phi) \bar{w}^{2}+(320 \phi s(1-\bar{y})+1+\phi) \bar{w}-4+4 \bar{y}=0 .
$$




\section{References}

[1] Azevedo Araujo, R. \& Moreira, T. B. S. (1994) A dynamic model of prodnction and traffic of drugs. Econ. Lett. 82, 371-376.

[2] BaLl. P. (2004) The physical modelling of human social systems. ComPlexUs 1, 190-206.

[3] Becker, G. S. (1968) Crime and punishment: An economic approach. J. Political Econ. 76, $168-217$.

[4] Beckfr, G. S. (1993) Nobel lecture: The economic way of looking at behavior. J. Political Econ. 101, 385-409.

[5] Brandt, H., Hauert, C. \& Sigmund, K. (2006) Punishing and abstaining for public goods. PNAS 103, 495-497.

[6] Campbell, M. \& Ormerod. P. (1998) Social interactions and the dynamics of crime. Accessed April 2010. URL: http://www.volterra.co.uk/publications/04/crime.pdf

[7] Carpenter, J. P. (2007) The demand for punishment. J. Econ. Behav. Organ. 62, 522-542.

[8] Fehr, E. \& GaChTER, S. (2000) Cooperation and punishment in public goods experiments. Am. Econ. Rev. 90, 980-994.

[9] Feichtinger, G., Grienauer. W. \& Tragler, G. (2002) Optimal dynamic law euforcement. Eur. J. Oper. Res. 141, 58-69.

[10] Grass. D.. Caulkins. J. P. , Feichtinger, G., Tragler. G. \& Behrens. D. A. (2008) Optimlal Control of Nonlinear Processes, with Applications in Drigs, Corription and Terror. Springer Verlag, Berlin.

[11] Henrich, J., Mc:Elreath, R., Barr, A., Ensminger, J., Barrett, C., Bolyanatz, A., Cardenas, J. C. . Gurven, M.. Gwako. E., Henrich. N., Lesorogol. C., Marlowe, F., Tracer, D. \& ZIKER. J. (2006) Costly punishment across human societies. Science 312, 1767-1770.

[12] Hirsch. M. W. \& SMale, S. (1974) Differential Equations. Dymanical Sustems and Linear Algebra. Academic press, New York.

[13] Johnson, D., Stopka, P. \& KNights, S. (2003) The puzzle of hnman cooperation (Brief communications). Nature 421, 911-912.

[14] MaY, R. M. (2001) Stability and Complexity in Model Ecosystems. Princeton University Press, New Jersey.

[15] Meacci. L. \& NuÑo, J. C. (2010) Spatially distributed W-models (In preparation).

[16] NuÑo, J. C., Herrero, M. A. \& Primicerio, M. (2008) A triangle model of criminality. Physica A 387, 2926-2936.

[17] NuÑo, J. C., Herrero, M. A. \& Primicerio, M. (2010) A mathematical model of a criminalprone society. Discrete Continuous Dym. Syst. - $S$ (in press).

[18] Ormerod, P. Mounfield. C. \& SMIth, L. (2001) Non-Linear Modelling of Burglary and Violent Crine in the UK. Volterra Consulting Ltd.

[19] Pierazzini, S. \& NuÑo, J. C. (2008) Mathematica Dentonstration. Accessed April 2010. URL: http://demonstrations. wolfram.com/ATriangleModelOrCriminality/

[20] ReED. W. J. (2001) The Pareto, Zipf and other power law. Economics lett. 74, 15-19.

[21] Sмгтн, D. A. (1977) Human population growth: Stability or explositon? Math. Mag. 50, $186-197$.

[22] VARGo. L. G. (1966) A note on crime control. Bull. Math. Biophys. 28, 375-378

[23] YU, P. L. (1973) A class of solntions for gronp decision problems. Manage. Sci. 19, 936-946.

[24] ZELENY, M. (1974) A concept of compromise solutions and the method of the displaced ideal. Comput. Oper. Res. 1, 479-496.

[25] Zhao, H., Zhilan, F. \& Castillo-Chavez, C. (2002) The dynamics of poverty aud crime. MTBI-02-08M. 